\title{
Evaluation of an Educator-Delivered School-Based Stress Management Program for Adolescents
}

\author{
Dana Carsley, Jessica Mettler, Amy J. Shapiro, Devin J. Mills, Elana L. Bloom,
} and Nancy L. Heath

\begin{abstract}
This study sought to determine if educators could deliver StressOFF Strategies (SOS), a brief classroombased stress management program (Shapiro \& Heath, 2013) to students and to evaluate educators' perspectives on SOS delivery. Seventeen educators participated in a two-day training and delivered the program to 555 adolescents $\left(51.4 \%\right.$ female; $M_{\text {age }}=14.45$ years, $\left.S D=0.74\right)$. Student evaluations revealed equivalent student satisfaction with educator and SOS team delivery on students' program rating; furthermore, $100 \%$ of educators strongly agreed $(38.5 \%)$ or agreed $(61.5 \%)$ that SOS was relevant, met their expectations, and was feasible within their school. Implications for schools and educators will be discussed.
\end{abstract}

\section{Evaluation of an Educator-Delivered School-Based Stress Management Program for Adolescents}

Adolescents are reporting high levels of stress (APA, 2014). Stress occurs when individuals can no longer cope with the demands of their environment and they perceive the environment as a threat to their wellbeing (Lazarus \& Folkman, 1984). High school is considered to be a critical period of academic and societal stress for adolescents as they begin to make their own decisions about their future, and experience various physical, psychological, and social changes (de Anda et al., 2000; Foret et al., 2012; Hankin, Mermelstein, \& Roesch, 2007). Research has demonstrated that the experience of stress in adolescents is often associated with negative outcomes (Byrne, Davenport, \& Mazanov, 2007; Kraag, Zeegers, Kok, Hosman, \& Abu-Saad, 2006; Rudolph \& Hammen, 1999), such as depression (Compas, Orosan, \& Grant, 1993; Galaif, Sussman, Chou, \& Wills, 2003), anxiety (Byrne et al., 2007; Rudolph, 2002), suicidal ideation (Diaz, Symnatov, \& Rickert, 2002), as well as poor academic performance (Frydenberg et al., 2004; Kaplan, Liu, \& Kaplan, 2005). Given the psychological and physical implications of stress (Frydenberg et al., 2004; Hankin et al., 2007), it is essential for adolescents to receive accessible and effective stress management knowledge and strategies in order to help them cope.

When individuals perceive that the demands of the situation are greater than their ability to cope, they can experience cognitive, behavioural, and/or emotional symptoms of stress (Washington, 2009). Stress has been shown to be particularly prevalent during the high school years as students create and interact with their social environments, form their personal identities, and go through physical and psychological changes (Foret et al., 2012; Hankin et al., 2007). Furthermore, high school represents a period in which 
adolescents also report experiencing specific in-school stressors related to academics such as the concern over grades, and present and future performance (de Anda et al., 2000). In a recent report by the American Psychological Association (2014), "Stress in America: Are Teens Adopting Adults' Stress Habits?" over 1000 adolescents participated in an online survey in which they were asked about their attitudes and perceptions of stress and stress management. According to the survey, $83 \%$ of adolescents reported school as the most common stressor; one which can negatively affect grades, time management, and personal relationships (APA, 2014). Adolescents have also indicated that they experience stress about the future (APA, 2014; de Anda et al., 2000). Although adolescents are reporting high school-related stress, the majority revealed they did not know how to manage their stress; in fact, they were reportedly unaware if they were adequately managing their stress and did not make enough time for stress management. In addition, results from the survey showed that adolescents experienced difficulties finding examples of healthy coping and stress management (APA, 2014).

According to Foret and colleagues (2012), there is a need for interventions that focus on stress management and stress reduction for adolescents. Stress management training can support adolescents in building resilience by limiting worries and perceived stress, enhancing self-esteem, and encouraging healthy coping (Foret et al., 2012). Furthermore, research has indicated that adolescents' ability to manage and cope with stress can positively affect their current and future psychological well-being (Compas et al., 1993; Compas, Connor-Smith, Saltzman, Thomsen, \& Wadsworth, 2001).

The school has been suggested as an important setting to build resilience in youth and limit the negative long-term psychological effects and consequences associated with stress (Frydenberg et al., 2004). Schools allow access to a large number of individuals and have been shown to reduce the stigma often associated with stress as students are not singled out (Fridrici \& Lohaus, 2009; Shochet et al., 2001). Additionally, due to the fact that the majority of students can be reached in this setting, participation and retention rates are not a concern (Fridrici \& Lohaus, 2009; Huberty, 2012; Shochet et al., 2001), and expenses, and organizational and transportation needs are minimized (Frydenberg et al., 2004; Lock \& Barrett, 2003). Given that adolescents frequently report school-related stressors (APA, 2014; de Anda et al., 2000), it would be important to address stress in youth and implement stress management programs directly in the classroom setting, as opposed to students being pulled out or seeking limited community resources.

Although many school-based stress management programs exist for children and adolescents (e.g., Bothe, Grignon, \& Olness, 2014; Bunn, Bifulco, Lorenc, \& Robinson, 2007; Garcia, Kemmick Pintor, \& Lindgren, 2010; Gelkopfl \& Berger, 2009; Hampel, Meier, \& Kummel, 2008; Kraag, Van Breukelen, Kok, \& Hosman, 2009; Szabo \& Marian, 2012), schools often find these programs lengthy, timeconsuming, or difficult to implement (Fridrici \& Lohaus, 2009). For instance, in a meta-analysis of schoolbased stress management programs implemented between 1976 and 2003, Kraag and colleagues (2006) tentatively concluded that primary prevention school-based stress management training programs that focus on promoting mental health were most effective. However, when examining these programs more closely, the sessions for each program were often implemented multiple times per week, and the majority of the programs required outside personnel (e.g., mental health professional, graduate student, 
undergraduate student, experimenter) to deliver or assist in delivering the program with the classroom teacher. Although these programs appeared to be effective, schools often reject programs that are difficult to integrate within their schools, such as programs that are time-consuming and require additional outside personnel to deliver the programs (Fridrici \& Lohaus, 2009).

A solution for decreasing the demands on schools while increasing limited resources would be to have educators deliver the stress management programs in their classrooms. According to Frydenberg and colleagues (2004), school-based programs are successful when the teacher is actively involved in the delivery of the program. In their study, "The Best of Coping: Developing Coping Skills Program" (Frydenberg \& Brandon, 2002) was delivered four separate times in two school settings, which comprise four studies. In Study 1, a psychologist or counsellor delivered the program to 83 adolescents (53\% female), and in Study 2, the first and third author delivered the program to 113 adolescents (49.5\% female; Frydenberg et al., 2004). In Study 3, there were 88 adolescents ( $44 \%$ female); 43 students received the program delivered by a team that consisted of a teacher and psychologist, and 45 students served as the control group. Similarly, in Study 4, a teacher and psychologist team delivered the program to 235 adolescents (45\% female); 179 students received the intervention and 56 students were the control group. In Study 4, the teachers received a condensed version of the training to deliver this program compared to a more complete and thorough training that the teachers in Study 3 received. The program included an examination of coping strategies, problem solving, communication, decision-making, goal setting, and time management. Overall, the four studies demonstrated modest support for the enhancement of stress coping skills; however, the program was shown to be most effective in Study 3, when it was delivered collaboratively by the psychologist and classroom teachers that were more thoroughly trained in program delivery compared with the limited training teachers in Study 4 received. Although mental health prevention in the school is typically assumed to be the role of the school psychologist, results revealed that when teachers are involved in the training and delivery of the program, the program could be even more beneficial (Frydenberg et al., 2004).

In a more recent study involving educators as deliverers, Hampel and colleagues (2008) examined the effectiveness of Anti-Stress-Training (AST), a school-based universal stress management prevention program for adolescents. In this study, 17 teachers were coached on how to deliver the AST program to 320 students ( $50 \%$ female) during class; 138 adolescents received the AST program, and 182 adolescents served as no-treatment controls. Teachers were trained by doctoral students and received information on stress, relaxation exercises, experiential education, an information booklet, and techniques for integrating stress management within the curriculum. Following the delivery of the six-week AST program, teachers were asked to assess the training they experienced. The majority of teachers indicated that students' selfefficacy was enhanced, and $57 \%$ of teachers reported that the training was useful. In addition to the teacher reports, students rated the training as highly acceptable. This study demonstrates how a universal stress management program could be beneficial for students when delivered by a classroom educator. Nevertheless, the time required to implement a six-week program is a challenge for many schools. Given the emphasis on academic performance in schools, one of the concerns school staff have when 
implementing new programs is the loss of time that is typically devoted to curriculum (Fridrici \& Lohaus, 2009).

Researchers have also investigated whether students prefer an educator-delivered school-based program that took place after school hours. Garcia and colleagues (2010) assessed the feasibility and acceptability of a 14-week school-based coping intervention, "Project Wings," for Latina adolescents that consisted of two interventions; the first intervention included 10 participants and took place during school hours, and the second intervention included 11 participants and occurred after school. This school-based coping program was co-led by an experienced facilitator and a school staff member and consisted of weekly meetings that included sharing circles, relaxation exercises, and skill building. Feedback from the students indicated overall acceptability and program satisfaction, and the authors noted that having a school-based facilitator ensured consistency and increased connectedness with the students. Although the students reported mixed results regarding their preference for the scheduling of the program (after school vs. during school hours), similar to the abovementioned stress management programs, this program was lengthy and required consistent support from outside personnel.

To address the limitations of the stress management programs reviewed earlier, StressOFF Strategies (SOS; Shapiro \& Heath, 2013) was created as a brief, universal single-session school-based stress management program for adolescents. SOS is a 45-minute stress management program that focuses on effective coping strategies to help adolescents manage their stress. The program consists of four key components: (a) Psychoeducation, (b) Decreasing stigma around experienced stress and associated mental health problems, (c) Teaching and practicing coping skills, and (d) Follow-up reinforcement of skills. In order to evaluate the preliminary feasibility and social acceptability of this program, trained SOS team members delivered SOS to over 565 adolescents (57\% female), and demonstrated positive post-program results (Shapiro, Heath, \& Carsley, 2015). SOS was found to be teen-friendly, highly engaging, and adolescents reported that they were willing to use the learned strategies in the future. Specifically, following the single-session program, $88.7 \%$ of students rated SOS as good to excellent, and $86.5 \%$ of students indicated that they learned a medium amount to a lot overall (Shapiro et al., 2015). Furthermore, $80.5 \%$ to $84.1 \%$ of participants indicated that they understood the strategies taught quite well to very well, and $71.0 \%$ to $82.5 \%$ were willing to use these strategies in the future. Findings from this program have been published in academic journals and presented at academic conferences (Shapiro \& Heath, 2013; Shapiro \& Heath, 2014; Shapiro et al., 2015; Shapiro, Mettler, Carsley, Hu, \& Heath, 2014; Shapiro, Hu, Joly, Emery, \& Heath, 2013).

Consistent with the research reviewed above, results from the SOS program demonstrate that adolescents need and are willing to participate in stress management training. Research has shown that stress management programs are particularly effective when classroom educators have integral roles in the delivery of the program (e.g., Frydenberg et al., 2004; Hampel et al., 2008); however, existing educatordelivered stress management programs for adolescents often require multiple sessions over longer periods of time. Although SOS demonstrated positive post-program results when delivered by trained SOS team members, outside delivery of stress management programs and the increasing demands on school mental health resources are not always feasible with limited school budgets and time constraints (Evans \& Weist, 
2004). In order to address these challenges, it would be beneficial for a universal, brief single-session school-based stress management program in schools that is both cost-effective and sustainable over time to be exclusively delivered by existing school personnel.

The present study sought to determine if educators (classroom teachers and technicians) could deliver SOS to their students and to determine educators' perspectives on SOS delivery. Specific objectives were to 1 ) assess the feasibility and social acceptability of SOS when delivered by educators through students' post-program reports of learning, program satisfaction, understanding of and willingness to use the stress management strategies taught, 2) compare student evaluations of educator versus SOS team program delivery and 3) assess educators' overall program satisfaction, knowledge of material, and perspectives on the program's feasibility.

\section{Method}

\section{Summary of Program}

StressOFF Strategies is a brief, universal, single-session school-based stress management program for adolescents consisting of four key components: (a) Psychoeducation, (b) Decreasing stigma, (c) Coping skills, and (d) Follow-up. In the psychoeducation component of the program, students are taught about stress and its psychological, physical, and behavioural characteristics. In order to decrease stigma, students are informed on the universality of stress through video clips and a "Celebrity Stress Trivia" game. The coping skills that students are taught include a combination of cognitive behavioural techniques (e.g., cognitive restructuring, progressive muscle relaxation) and mindfulness-based techniques (e.g., present-moment awareness and acceptance) that have been found to be effective for stress management. Finally, students are provided with a pamphlet and resources for follow-up and reinforcement of skills.

\section{Participants}

The sample of educators consisted of 14 teachers and technicians (78.57\% female) from nine high schools in Montreal, Quebec, Canada, representing low to high socioeconomic status. Although 17 educators were trained to deliver the SOS program, three educators were unable to deliver the program due to classroom scheduling issues. Over a period of two months, the remaining 14 educators delivered the program in their classrooms or in an assembly setting to a total of 631 students. Of these participants, 76 students were excluded from analyses for two reasons. First, due to scheduling, some of the sessions had specific time constraints; as such, a number of students did not have enough time to complete the post-program measure, and were excluded from the final analyses. Second, the majority of the students excluded represented an unusual population that the program was not designed for; specifically, these students were from an alternative school, had moderate to severe emotional and behavior disabilities, and many demonstrated severe learning disabilities. Although the educator attempted to deliver SOS to this group of students, it was discovered early on that the material designed 
for this program was the wrong level for these students. As a result, the final sample comprised a total of 555 students $(51.4 \%$ female; Mage $=14.45, S D=0.74)$.

\section{Measures}

Student evaluations. After participating in the SOS program, students were asked to evaluate their educator's delivery of the program. Using four-point Likert-type scales, students rated how much they felt they had learned on a scale ranging from nothing (0) to a lot (3); the complexity of the program on a scale from not sure (0) to too complicated (3); and the overall program on a scale from poor (1) to excellent (4). The students were also asked to evaluate their understanding of the strategies taught in the program (thought challenge, muscle relaxation, self-observer, and support and better choices) using a four-point Likert scale ranging from not very well (1) to understand very well (3), with an option for already knew (4). Finally, they were asked to rate their willingness to use these strategies in the future on a five-point Likert scale ranging from never: I don't need to use stress management (0) to a/ways (4).

Educator evaluations. The educators were asked to evaluate their experience with SOS by rating a number of items on a five-point Likert scale ranging from strongly agree or always (1) to strongly disagree or never (5). This questionnaire consisted of 17 items assessing their satisfaction with SOS (e.g., "I found the program to be beneficial"), their knowledge of the material provided (e.g., "I now know how to deliver the StressOFF program"), and their perspectives on the program feasibility (e.g., "I found the StressOFF program to be feasible in my school").

\section{Procedure}

Following university ethics and school board approval, the educators were trained to deliver the SOS program during a two-day workshop offered by the SOS team. During this training session, the educators were first shown a live presentation of the SOS program by a trained research assistant. The educators then received a manual with a detailed script of the program to guide their delivery of the SOS program to their students and the primary researcher provided an in-depth explanation of the four strategies in the program. At the end of the first day of the workshop, the educators were asked to practice the presentation at home. The second day of the workshop consisted of a group presentation, in which the educators took turns practicing and presenting either on their own or in pairs, and received feedback from the primary researcher.

Over the following eight weeks, the educators distributed consent forms to students in their schools, so that the students could participate in the evaluation of the educators' presentation of SOS. Following consent form returns, the educators scheduled times in which they would deliver SOS to their students. For each presentation, trained research assistants went to the school to deliver assent forms and questionnaires for the students to fill out before and after the presentation. 
Two months following the initial two-day workshop, a half-day follow-up session was conducted in which the educators received feedback on their program delivery and had the opportunity to evaluate their experience with SOS training and delivery.

\section{Results}

When examining normality for each item within the student evaluations of the educators and of the SOS team members, no departure from normality was found. Furthermore, no outliers were identified in the data. However, students who had skipped more than two items on the student evaluations, as well as those who had not indicated a gender, were excluded from analyses.

\section{Feasibility and Social Acceptability of a Stress Management Program Delivered by Educators}

In the post-program reports, students were asked to rate the amount of information they learned about stress management and their overall program satisfaction. Following the program, $80 \%$ of students indicated that they learned a medium amount to a lot $(M=2.00, S D=0.70)$ on a four-point scale from nothing (0) to a lot (3). In terms of program satisfaction, the majority of students (83.2\%) rated the program as good to excellent $(M=3.05, S D=0.68)$ on a four-point scale from poor (1) to excellent (4).

Students were also asked to rate their understanding of the stress management strategies taught and willingness to use these strategies in the future. Overall, the majority of students reported high understanding of and willingness to use all four of the stress management strategies taught (see Figures 1 and 2).

\section{Comparison of Educators vs. SOS Team Delivery of a Stress Management Program}

In order to compare student ratings of educator versus SOS team program delivery, a 2 X 2 ANOVA was conducted for each item on the questionnaire. With a total of 10 ANOVAs, the alpha was modified to .005 using the Bonferroni method. As displayed in Table 1, student evaluations of the educators' program delivery were similar for both groups of presenters (educators and SOS team members). Although there was a significant difference on the amount students reported to have learned $(F(1,1113)=9.629$, $p=.002 ; \eta=.009)$ and their reports of program satisfaction $(F(1,1062)=8.323, p=.004 ; \eta=.008)$ with SOS team program delivery being rated as slightly higher, these differences were marginal. No significant differences in male and female student evaluations for the educators compared to the SOS team members were found. 


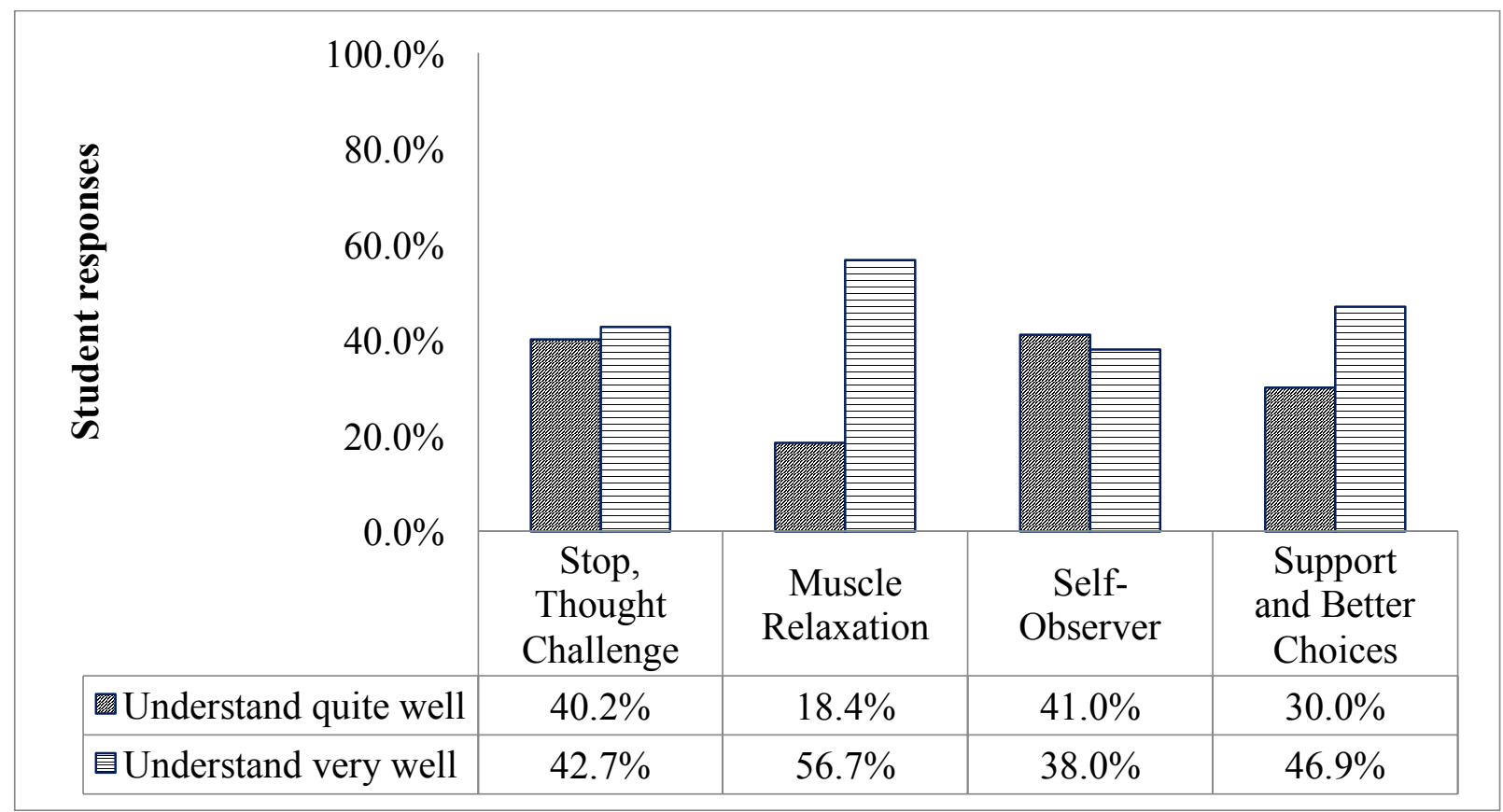

Fig. 1. Students' understanding of stress management strategies taught: Percentage of students indicating that they understood quite well and very well the four strategies presented in StressOFF Strategies.

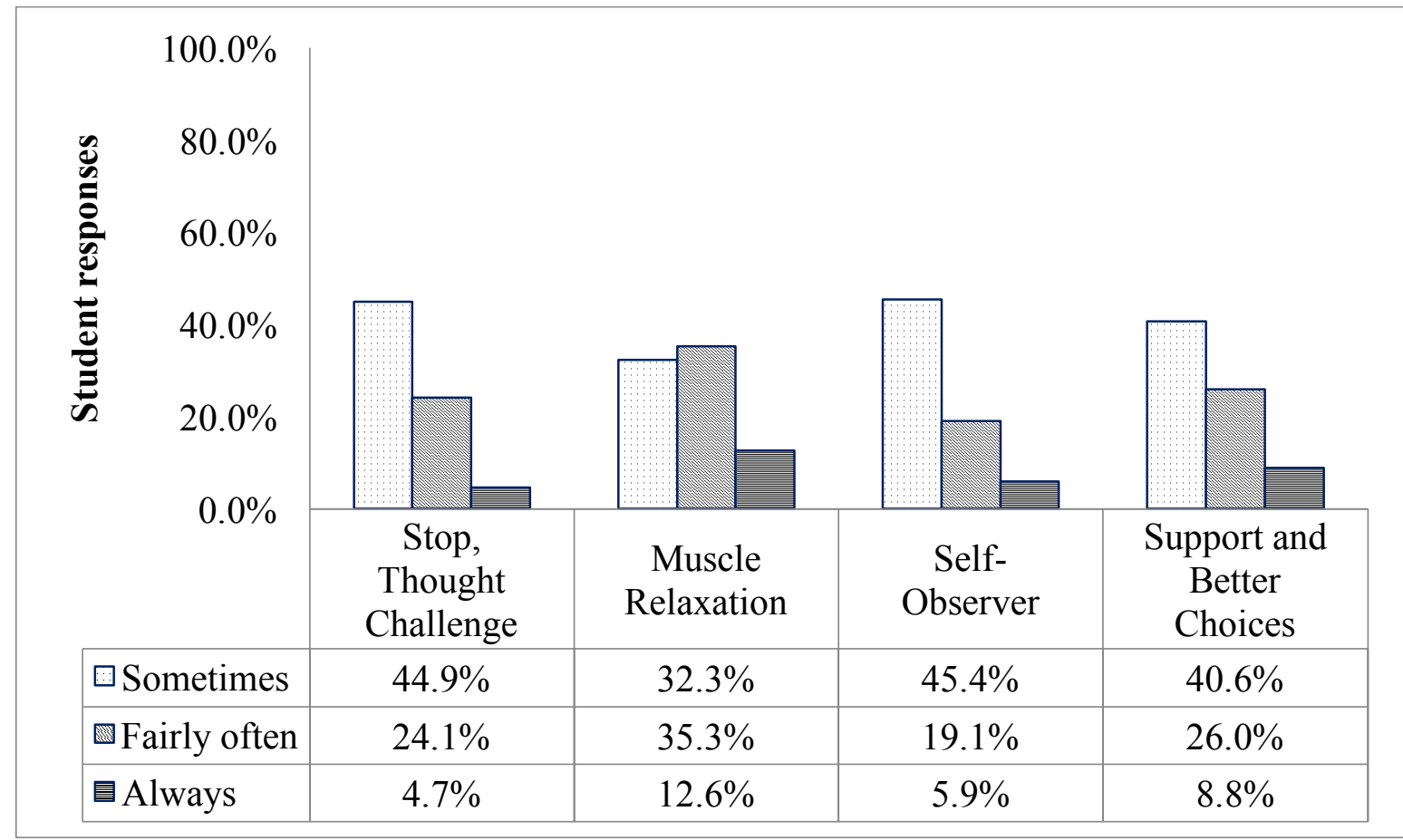

Fig 2. Students' willingness to use the stress management strategies taught: Percentage of students indicating that they were sometimes, fairly often, and always willing to use the four strategies presented in StressOFF Strategies in order to deal with future stress. 


\section{Table 1}

Student evaluations of StressOFF Strategies when the program was delivered by educators and the SOS team, and comparison of delivery between the two groups.

\begin{tabular}{|c|c|c|c|c|c|c|c|c|}
\hline & \multicolumn{2}{|c|}{$\begin{array}{l}\text { Students from } \\
\text { educator delivery }\end{array}$} & \multicolumn{2}{|c|}{$\begin{array}{l}\text { Students from SOS } \\
\text { team delivery }\end{array}$} & \multicolumn{4}{|c|}{ Group Differences } \\
\hline & $M$ & $S D$ & $M$ & $S D$ & $d f$ & $\boldsymbol{F}$ & $\eta^{2}$ & $p$ \\
\hline $\begin{array}{l}\text { Amount students learned abol } \\
\text { management after workshop }{ }^{1}\end{array}$ & 2.01 & 0.7 & 2.14 & 0.661 & 1 & 9.629 & .009 & $.002^{*}$ \\
\hline Program satisfaction ${ }^{2}$ & 3.04 & 0.684 & 3.16 & 0.649 & 1 & 8.323 & .008 & $.004^{*}$ \\
\hline \multicolumn{9}{|c|}{$\begin{array}{l}\text { Understanding of stress management } \\
\text { strategies }^{3}\end{array}$} \\
\hline Thought challenge & 2.51 & 0.769 & 2.53 & 0.754 & 1 & 0.093 & .000 & .760 \\
\hline Muscle relaxation & 2.92 & 0.758 & 2.88 & 0.662 & 1 & 1.144 & .001 & .285 \\
\hline Self-observer & 2.42 & 0.815 & 2.88 & 0.662 & 1 & 0.096 & .000 & .756 \\
\hline Support and better choices & 2.69 & 0.822 & 2.44 & 0.766 & 1 & 0.213 & .000 & .644 \\
\hline \multicolumn{9}{|c|}{$\begin{array}{l}\text { Willingness to use stress management } \\
\text { strategies }^{4}\end{array}$} \\
\hline Thought challenge & 1.98 & 0.987 & 1.93 & 0.943 & 1 & 1.370 & .001 & .242 \\
\hline Muscle relaxation & 2.32 & 1.096 & 2.38 & 1.037 & 1 & .196 & .000 & .658 \\
\hline Self-observer & 1.91 & 1.0146 & 1.97 & 0.974 & 1 & .326 & .000 & .568 \\
\hline Support and better choices & 2.09 & 1.075 & 2.18 & 1.028 & 1 & .713 & .001 & .399 \\
\hline
\end{tabular}

* Significant at the modified alpha level $(p<.005)$

${ }^{1}$ Likert-type four-point scale $(0=$ Nothing at all; $1=$ Small amount; $2=$ Medium amount; $3=$ A lot $)$

${ }^{2}$ Likert-type four-point scale ( 1 = Poor; 2 = Satisfactory; 3 = Good; 4 = Excellent $)$

${ }^{3}$ Likert-type four-point scale $(1=$ Not very well; $2=$ Understand quite well; 3 = Understand very well; $3=$ Already knew)

${ }^{4}$ Likert-type five-point scale $(0=$ Never - don't need to use stress management; $1=$ Never - don't like this technique; 2 = Sometimes; 4 = Always) 


\section{Educator Perspectives on Delivering a Stress Management Program}

Program satisfaction. When asked how satisfied they were with SOS, 100\% of educators strongly agreed $(38.5 \%)$ or agreed $(61.5 \%)$ that SOS was relevant and met their expectations and $100 \%$ of educators strongly agreed (46.2\%) or agreed (53.8\%) that SOS was beneficial. Similarly, $100 \%$ of educators strongly agreed $(61.6 \%)$ or agreed $(38.5 \%)$ that SOS was a valuable professional/personal development experience, and $100 \%$ of educators strongly agreed $(53.8 \%)$ or agreed $(46.2 \%)$ that they would recommend SOS to other colleagues.

Knowledge of material. In addition to overall program satisfaction, educators were also asked to rate their knowledge of SOS material. Results revealed that $92.3 \%$ of educators strongly agreed (38.5\%) or agreed $(53.8 \%)$ that they now know how to deliver SOS and are comfortable with delivering the introduction section of the program. Furthermore, $100 \%$ of educators strongly agreed $(30.8 \%)$ or agreed $(69.2 \%)$ that they are comfortable with delivering the psychoeducation section of the program, and $92.3 \%$ of educators strongly agreed $(30.8 \%)$ or agreed $(61.5 \%)$ that they are comfortable with delivering the strategies in the program. Overall, $92.3 \%$ strongly agreed $(30.8 \%)$ or agreed $(61.5 \%)$ that they are confident in their ability to deliver SOS.

Feasibility. Finally, $100 \%$ of educators strongly agreed (38.5\%) or agreed $(61.5 \%)$ that this program was feasible within their schools.

\section{Discussion}

The purpose of this study was to assess the feasibility and social acceptability of educators' delivery of StressOFF Strategies, a brief, universal single-session school-based stress management program, to their students and to determine educators' perspectives on program delivery. Specifically, the aims of this study were to 1) evaluate the feasibility and social acceptability of SOS when delivered by educators through students' post-program reports of learning, program satisfaction, understanding of and willingness to use the stress management strategies taught, 2) compare student evaluations of educator versus SOS team program delivery, and 3) assess educators' overall program satisfaction, knowledge of material, and perspectives on the program's feasibility.

The first objective of this study was to assess the feasibility and social acceptability of educators' delivery of SOS through students' post-program reports. Following the educators' delivery of the program, the majority of students indicated that they learned a medium amount to a lot about stress and stress management. Furthermore, the majority of students rated the SOS program as good to excellent. When asked about their understanding of the stress management strategies taught and their willingness to use these strategies in the future, the majority of students indicated that they understood the strategies quite well to very well, and that they were willing to use these techniques sometimes to always. Based on these high student evaluations, these findings support the feasibility and social acceptability of SOS when delivered by educators. 
Many existing stress management programs are lengthy, time consuming, and require additional outside personnel to deliver or assist in the delivery of the program (Fridrici \& Lohaus, 2009; Kraag et al., 2006). Furthermore, educators often indicate that they are reluctant to address mental health resiliency in the classroom due to their lack of training, time, and skills (Ekornes, 2015; Reinke, Stormont, Herman, Puri, \& Goel, 2011; Stormont, Reinke, \& Herman, 2011). These results show that with a small amount of training, educators can deliver a brief school-based stress management program and encourage mental health resilience for their students in their classrooms.

The second objective of this study was to determine if educators' delivery of SOS was comparable with the original delivery of the program by SOS team members. Overall, results from the student evaluations of the educators' program delivery and the SOS team members' program delivery were similar for both groups of presenters. These results demonstrate that the educators were as successful as trained professionals in delivering a stress management program. Although there was a significant difference for the amount students had learned following the program and the students' rating of the program, these differences were minor. Specifically, $80 \%$ of students indicated that they learned a medium amount to a lot following the educators' delivery of the program, compared to $86.5 \%$ of students following the SOS team's delivery of the program. Furthermore, $83.2 \%$ of students rated the program as good to excellent when it was delivered by the educators, compared to $88.7 \%$ of students following the SOS team's delivery of the program. Consistent with previous literature, when educators are involved in the delivery of the program, the program is well received by students (Frydenberg et al., 2004). As such, these results indicate that educators are able to deliver a brief stress management program as successfully as outside professionals. Interventions for mental health resilience are typically considered to be the role of the mental health professional (e.g., school psychologists); however, these findings suggest that educators can assist mental health professionals in supporting the mental health needs for their students. For instance, educators can lead in promoting and enhancing student well-being through the incorporation of universal stress management programs within their classroom curriculum.

The final objective of this study was to assess educators' overall program satisfaction, knowledge of material, and perspectives on the program's feasibility. Results revealed that all of the educators strongly agreed or agreed that 1) SOS was relevant and met their expectations, 2) the SOS program was beneficial, 3) the SOS training was a valuable professional/personal development experience, and 4) they would recommend SOS to their colleagues. These findings suggest that educators responded positively to their experience in the training of the program.

When asked about their knowledge of material at the follow-up session, the majority of educators strongly agreed or agreed that a) they now know how to deliver SOS, b) they are now comfortable delivering SOS, and c) they are now confident in their ability to deliver the program. These findings imply that with minimal training, educators are able to deliver a brief stress management program to their students. Although many educators have indicated that dealing with mental health problems within their classrooms is a challenge due to minimal skills, training, and insufficient knowledge (Reinke et al., 2011; Stormont et al., 2011), they have also reported that teachers are in an important position in which they can support the mental health needs of their students (Ekornes, 2015). Teachers have also indicated that 
mental health promotion should be part of their professional role, particularly when implementing classroom behavioural interventions (Graham, Phelps, Maddison, \& Fitzgerald, 2011; Reinke et al., 2011). These findings suggest that if educators are provided with the skills to implement a school-based stress management program, they might be more confident, comfortable, and willing to support students' mental health needs in their classrooms.

Finally, educators were asked to report on the feasibility of this program within their school. All of the educators strongly agreed or agreed that SOS was feasible to implement in their school. As previously mentioned, schools often reject programs that are too demanding or time-consuming due to other school and curricular priorities (Fridrici \& Lohaus, 2009; Graham et al., 2011). Therefore, these findings suggest that a less demanding stress management program within the schools can be implemented without disrupting other school commitments.

Despite these findings, there are some limitations that must be considered. Although there were no significant differences in the students' understanding of the strategies taught when delivered by educators and the SOS training team, educators reported that they experienced difficulties explaining the mindfulness-based stress management strategy to their students. Certain strategies can be more easily learned and disseminated to students (e.g., muscle relaxation), however, mindfulness-based strategies may be more difficult to understand and teach. Anecdotally, it was revealed in follow-up discussions that one third of the educators incorporated relaxation exercises in place of the present-moment awareness inherent to mindfulness. In future studies and in efforts to continue educator-delivered stress management programs, a complex and novel notion such as mindfulness may require more time to train, and a targeted evaluation of program fidelity should be included to ensure that the strategy is implemented correctly. Additionally, the focus of the present study was to evaluate the feasibility and social acceptability of SOS as delivered by educators compared to SOS as delivered by SOS team members, but the current design would be enhanced by the inclusion of a comparable control intervention. Although it would have been beneficial to ensure that the samples were equivalent, many of the same schools and regions participated in both studies, demonstrating that these samples are comparable. Finally, one educator from an alternative school for at-risk youth experienced difficulties presenting the SOS program to the students and suggested that the program should be adapted for this specific population. It would be important for future implementations of stress management programs to ensure that the program that is being implemented is relevant for different student populations.

The present study demonstrated the feasibility and social acceptability of an educator-delivered universal brief single-session school-based stress management program for adolescents. The results of this study suggest that it is not essential for an outside presenter or mental health professional (e.g., school psychologist, counsellor) to deliver a stress management program to adolescents; educators can include brief programs such as these within their classrooms with minimal outside training.

The findings from this study have important implications for schools, educators, and teacher education. Primarily, including a stress management program delivered by educators within the school can decrease the cost of the program while still enabling the students to benefit from receiving training in stress 
management skills and strategies. Furthermore, educators can now assist school psychologists in supporting the mental health needs of their students. Due to the fact that students spend the majority of their time with their teachers, this study demonstrates that educators can also support students' mental health within their classrooms, in addition to their academics. Finally, the knowledge and skills in the area of stress management gained by the educators can be applied in other school contexts with students, as well as for their own personal benefit.

\section{References}

American Psychological Association. (2014). Stress in America 2014. Retrieved from https://www.apa.org/news/press/releases/stress/2013/stress-report.pdf

Bothe, D., Grignon, J. B., \& Olness, K. N. (2014). The effects of a stress management intervention in elementary school children. Journal of Developmental \& Behavioral Pediatrics, 35(1), 62-67.

Bunn, A., Bifulco, A., Lorenc, A., \& Robinson, N. (2007). Solutions on stress (SOS): Programmes, packages and products for helping teenagers. Young Consumers, 8(1), 29-35. doi:10.1108/17473610733758

Byrne, D. G., Davenport, S. C., \& Mazanov, J. (2007). Profiles of adolescent stress: The development of the adolescent stress questionnaire (ASQ). Journal of Adolescence, 30(3), 393-416. doi:10.1016/j.adolescence.2006.04.004

Compas, B. E., Connor-Smith, J. K., Saltzman, H., Thomsen, A. H., \& Wadsworth, M. E. (2001). Coping with stress during childhood and adolescence: Problems, progress, and potential in theory and research. Psychological Bulletin, 127, 87-127.

Compas, B. E., Orosan, P. G., \& Grant, K. E. (1993). Adolescent stress and coping: Implications for psychopathology during adolescence. Journal of Adolescence, 16, 331-349.

de Anda, D., Baroni, S., Boskin, L., Buchwald, L., Morgan, J., Ow, J., et al. (2000). Stress, stressors and coping among high school students. Children and Youth Services Review, 22(6), 441-463.

Diaz, A., Symantov, E., \& Rickert, V. I. (2002). Effect of abuse on health: Results of a National survey. Archives of Pediatric and Adolescent Medicine, 156, 811-817.

Ekornes, S. (2015). Teacher perspectives on their role and the challenges of inter-professional collaboration in mental health promotion. School Mental Health. doi:10.1007/s12310-015-9147-y

Evans, S. W., \& Weist, M. D. (2004). Commentary: Implementing empirically supported treatments in the schools: What are we asking? Clinical Child and Family Psychology Review, 7(4), 263-267.

Foret, M. M., Scult, M., Wilcher, M., Chudnofsky, R., Malloy, L., Hasheminejad, N., et al. (2012). Integrating a relaxation response-based curriculum into a public high school in Massachusetts. Journal of Adolescence, 35, 325-332. doi:10.1016/j.adolescence.2011.08.008

Fridrici, M., \& Lohaus, A. (2009). Stress-prevention in secondary schools: Online-versus face-to-facetraining. Health Education, 109, 299-313.

Frydenberg, E., \& Brandon, C. M. (2002). The best of coping. Melbourne: Oz Child. 
Frydenberg, E., Lewis, R., Bugalski, K., Cotta, A., McCarthy, C., Lucombe-Smith, N., et al. (2004). Prevention is better than cure: Coping skills training for adolescents at school. Educational Psychology in Practice: Theory, Research and Practice in Educational Psychology, 20(2), 117-134. doi:10.1080/02667360410001691053

Galaif, E. R., Sussman, S., Chou, C. P., \& Wills, T. A. (2003). Longitudinal relations among depression, stress, and coping in high risk youth. Journal of Youth and Adolescence, 32, 243-258.

Garcia, C., Kemmick Pintor, J., \& Lindgren, S. (2010). Feasibility and acceptability of a school-based coping intervention for Latina adolescents. The Journal of School Nursing, 26(1), 42-52. doi:10.1177/1059840509351021

Gelkopf, M., \& Berger, R. (2009). A school-based, teacher-mediated prevention program (ERASE-Stress) for reducing terror-related traumatic reactions in Israeli youth: A quasi-randomized controlled trial. Journal of Child Psychology and Psychiatry, 50(8), 962-971. doi:10.1111/j.1469-7610.2008.02021.x

Graham, A., Phelps, R., Maddison, C., \& Fitzgerald, R. (2011). Supporting children's mental health in schools. Teachers and Teaching, 17(4), 479-496. doi:10.1080/13540602.2011.580525

Hampel, P., Meier, M., \& Kummel, U. (2008). School-based stress management training for adolescents: Longitudinal results from an experimental study. Journal of Youth and Adolescence, 37, 1009-1024. doi:10.1007/s10964-007-9204-4

Hankin, B. L., Mermelstein, R., \& Roesch, L. (2007). Sex differences in adolescent depression: Stress exposure and reactivity models. Child Development, 78(1), 279-295.

Huberty, T. J. (2012). School-based interventions. In T. J Huberty (Ed.), Anxiety and depression in children and adolescents: Assessment, intervention and prevention. New York: Springer.

Kaplan, D. S., Liu, R. X., \& Kaplan, H. B. (2005). School related stress in early adolescence and academic performance three years later: The conditional influence of self expectations. Social Psychology of Education, 8, 3-17.

Kraag, G., Van Breukelen, G. J. P., Kok, G., \& Hosman, C. (2009). 'Learn Young, Learn Fair', a stress management program for fifth and sixth graders: Longitudinal results from an experimental study. Journal of Child Psychology and Psychiatry, 50(9), 1185-1195. doi:10.1111/j.1469-7610.2009.02088.x

Kraag, G., Zeegers, M. P., Kok, G., Hosman, C., \& Abu-Saad, H. H. (2006). School programs targeting stress management in children and adolescents: A meta-analysis. Journal of School Psychology, 44, 449-472. doi:10.1016/j/jsp.2006/07/001

Lazarus, R. S., \& Folkman, S. (1984). Stress, appraisal and coping. New York: Springer.

Lock, S., \& Barrett, P. M. (2003). A longitudinal study of developmental differences in universal preventive intervention for child anxiety. Behaviour Change, 20, 183-199.

Reinke, W.M., Stormont, M., Herman, K.C., Puri, R., \& Goel, N. (2011). Supporting children's mental health in schools: Teacher perceptions of needs, roles, and barriers. School Psychology Quarterly, 26(1), 1-13. doi:10.1037/a0022714

Rudolph, D. R. (2002). Gender differences in emotional responses to interpersonal stress during adolescence. Journal of Adolescent Health, 30, 3-13. doi:10.1016/S1054-139X(01)00383-4 
Rudolph, K. D., \& Hammen, C. (1999). Age and gender as determinants of stress exposure, generation, and reactions in youngsters: A transactional perspective. Child Development, 70(3), 660-667.

Shapiro, A. J., \& Heath, N. L. (2013). (c) StressOFF strategies: A stress management program for teens. Unpublished program and guide, Department of Educational and Counselling Psychology, McGill University, Montreal, QC.

Shapiro, A. J., \& Heath, N. L. (2014). Teen stress in our schools: A 45-minute program to improve coping skills. Education Canada, 54(2), 18-21.

Shapiro, A. J., Heath, N. L., \& Carsley, D. (2015). Effectiveness of a Brief School-Based Stress Management Program for Adolescents. Advances in School Mental Health Promotion.

Shapiro, A. J., Hu, T., Joly, M., Emery, A., \& Heath, N. L. (2013, June). Effectiveness of a brief schoolbased stress management program for adolescents. Poster session presented at the annual meeting of the Canadian Psychological Association, CPA, Quebec, QC.

Shapiro, A. J., Mettler, J., Carsley, D., Hu, T., \& Heath, N. L. (2014, March). Adolescents' perspectives on stress management strategy use following a brief school intervention. Poster presented at the annual convention of the Société Québécoise pour la Recherche en Psychologie, SQRP, Montreal, QC.

Shochet, I. M., Dadds, M. R., Holland, D., Whitefield, K., Harnett, P. H., \& Osgarby, S. M. (2001). The efficacy of a universal school-based program to prevent adolescent depression. Journal of Clinical Child Psychology, 30(30), 303-315.

Stormont, M., Reinke, W., \& Herman, K. (2011). Teachers' characteristics and ratings for evidence-based behavioral interventions. Behavioral Disorders, 37(1), 19-29.

Szabo, Z., \& Marian, M. (2012). Stress inoculation training in adolescents: Classroom intervention benefits. Journal of Cognitive and Behavioral Psychotherapies, 12(2), 175-188.

Washington, T. D. (2009). Psychological stress and anxiety in middle to late childhood and early adolescence: Manifestations and management. Journal of Pediatric Nursing, 24, 302-313. doi:10.1016/j.pedn.2008/04/011 


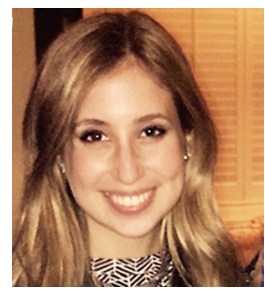

Dana Carsley, BEd, MEd, is an elementary school teacher and a PhD student in McGill University's Department of Educational and Counselling Psychology. Dana has worked as a teacher and special needs tutor for students at all levels. Her research focuses on classroom interventions to promote mental health, specifically teacher-led mindfulness and stress management programs. She has published several papers on mindfulness in educational settings, and has presented at numerous academic conferences and teacher workshops. She is a Tomlinson Scholar, and recipient of the Joseph-Armand Bombardier Canada Graduate Doctoral Scholarship from the Social Sciences and Humanities Research Council of Canada.

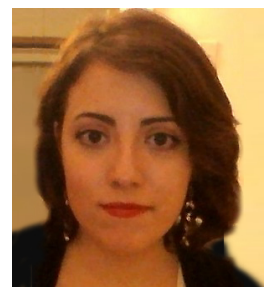

Jessica Mettler, BA, is a graduate student in the Human Development program at McGill University. Her research interests focus on the role of mindfulness in promoting mental health and well-being. She has experience in the development and evaluation of short mindfulness and stress management training programs for high school students, postsecondary students and mental health professionals. She has also published papers and presented at academic conferences and teacher workshops on stress management and mindfulness in educational settings. Finally, Jessica has been the recipient of a Master's Research Scholarship from the Fonds de recherche du Québec - Société et culture.

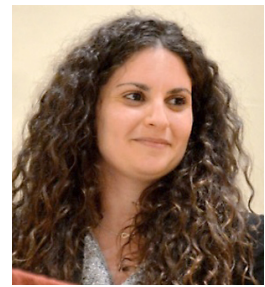

Amy Shapiro, BEd, MEd, is a secondary school teacher and is currently working on her $\mathrm{PhD}$ in Human Development in the Department of Educational and Counselling Psychology at McGill University. She developed the StressOFF Strategies program with Dr. Nancy Heath in 2013, which has since been delivered to thousands of students in English language school boards in Montreal and surrounding areas. Amy has published several papers on stress and other mental health issues in the schools, and has presented at various academic conferences and workshops. She is also a recipient of a doctoral scholarship from the Fonds de recherche du Québec - Société et culture.

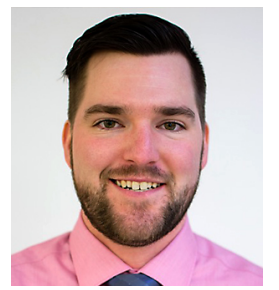

Devin J. Mills is a PhD Candidate at McGill University with an interest in applying Self-Determination Theory to the broad areas of adaptive and maladaptive functioning. His work currently surrounds problematic gaming, but has in the past included studies on non-suicidal self-injury and mindfulness within adolescent and young adult populations. He is a current member of the Development and Intrapersonal Resilience Research Team. 


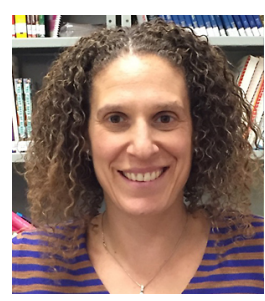

Elana Bloom, PhD, is a psychologist at the Lester B. Pearson School Board, having worked in elementary and high schools supporting students with mental health concerns and equipping staff to better understand mental health. Dr. Bloom is currently the Coordinator of the Family School and Support Treatment Team, a model in schools composed of behavioral technicians and consultants to support students with socioemotional and mental health concerns. She is also the Coordinator of the Center of Excellence for Mental Health team, where she provides workshops and builds capacity for school staff across the 10 English school boards in Quebec.

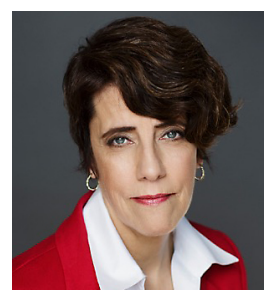

Nancy L. Heath, PhD, is a James McGill Professor in the Department of Educational and Counselling Psychology at McGill University. Her research centers on understanding and enhancing intrapersonal resilience in youth and young adults with a focus on factors related to emotion regulatory processes. She has published and presented extensively in this area and more broadly on issues pertaining to mental health in schools. Professor Heath conducts both basic and applied research, always with a strong knowledge translation and service emphasis. She has worked for over 25 years in partnership with schools to address mental health concerns in the schools. 